\title{
A real electro-magnetic placebo (REMP) device for sham transcranial magnetic stimulation (TMS) is
}

\author{
Simone Rossi ${ }^{\text {a,* }}$, Marisa Ferro ${ }^{\mathrm{a}}$, Massimo Cincotta ${ }^{\mathrm{b}}$, Monica Ulivelli ${ }^{\mathrm{a}}$, Sabina Bartalini ${ }^{\mathrm{a}}$, \\ Carlo Miniussi ${ }^{c, d}$, Fabio Giovannelli ${ }^{\text {b }}$, Stefano Passero ${ }^{\text {a }}$ \\ a Dipartimento di Neuroscienze, Sezione Neurologia, Università di Siena, Policlinico Le Scotte, Viale Bracci, I-53100 Siena, Italy \\ ${ }^{\mathrm{b}}$ Unità Operativa di Neurologia, Azienda Sanitaria di Firenze, Italy \\ ${ }^{\mathrm{c}}$ Unità di Neuroscienze Cognitive, IRCCS S. Giovanni di Dio FBF, Brescia, Italy \\ d Dipartimento di Scienze Biomediche e Biotecnologie, Università di Brescia, Italy
}

Accepted 8 November 2006

Available online 22 December 2006

\begin{abstract}
Objective: There is growing interest in neuropsychiatry for repetitive transcranial magnetic stimulation (rTMS) as a neuromodulatory treatment. However, there are limitations in interpreting rTMS effects as a real consequence of physiological brain changes or as placebo-mediated unspecific effects, which may be particularly strong in psychiatric patients. This is due to the fact that existing sham rTMS procedures are less than optimal. A new placebo tool is introduced here, called real electro-magnetic placebo (REMP) device, which can simulate the scalp sensation induced by the real TMS, while leaving both the visual impact and acoustic sensation of real TMS unaltered. Methods: Physical, neurophysiological and behavioural variables of monophasic and biphasic single-pulse TMS and biphasic 1 Hz and $20 \mathrm{~Hz}$ rTMS procedures (at different intensities) were tested in subjects who were expert or naïve of TMS. Results of the real TMS were compared with those induced by the REMP device and with two other currently used sham procedures, namely the commercially available Magstim sham coil and tilting the real coil by $90^{\circ}$.

Results: The REMP device, besides producing scalp sensations similar to the real TMS, attenuated the TMS-induced electric field (as measured by a dipole probe) to a biologically inactive level. Behaviourally, neither expert nor naïve TMS subjects identified the "coil at $90^{\circ}$ " or the "Magstim sham coil" as a real TMS intervention, whilst naïve subjects were significantly more likely to identify the REMP-attenuated TMS as real.

Conclusions: The "goodness of sham" of the REMP device is demonstrated by physical, neurophysiological, and behavioural results. Significance: Such placebo TMS is superior to the available sham procedures when applied on subjects naïve to TMS, as in case of patients undergoing a clinical rTMS trial.
\end{abstract}

(C) 2006 International Federation of Clinical Neurophysiology. Published by Elsevier Ireland Ltd. All rights reserved.

Keywords: TMS; rTMS; Placebo; Sham coil; Neuromodulation

\section{Introduction}

Born about 20 years ago as a tool to investigate the functionality of the corticospinal tract in intact humans, transcranial magnetic stimulation (TMS) is now increasingly employed in several fields of neuropsychiatry and neurosci-

\footnotetext{
Italian patent number: n. RM2006A000514.

* Corresponding author. Tel.: +390577 585 401; fax: +390577 270260 .

E-mail address: rossisimo@unisi.it (S. Rossi).
}

ence in general (Hallett, 2000; Rossini and Rossi, 2006). This is also due to technical advances which allow one to stimulate the brain repetitively. Such an approach is called repetitive TMS (rTMS), and represents, besides an established tool for demonstrating that brain regions are causally involved in a variety of cognitive functions (Walsh and Cowey, 2000; Pascual-Leone et al., 2000; Rossi and Rossini, 2004), even a therapeutic neuromodulatory strategy of increasing clinical relevance. The latter opportunity relies on the evidence that rTMS-induced excitability changes at 
cortical level may outlast the interventional time and that these modulatory effects may transiently improve symptoms of many neuropsychiatric diseases characterized by dysfunctions of regional excitability (Wasserman and Lisanby, 2001; Hoffman and Cavus, 2002; Fregni and Pascual-Leone, 2005).

However, both psychophysiological investigations and therapeutic trials with TMS require control conditions with sham (placebo) stimulation. Indeed, placebo effects induced by rTMS may be particularly relevant in neuropsychiatric patients (Wasserman and Lisanby, 2001) and even produce clearly detectable changes in brain activity, as recently demonstrated in parkinsonian patients (see Strafella et al., 2006).

None of the available sham conditions are fully satisfactory as to the absence of biological effects and failure to reproduce the scalp sensations induced by real TMS, especially concerning trigeminal afferents activation underneath the stimulation site (see later in the discussion). To obtain a more reliable sham stimulation, we developed the so-called REMP (real electro-magnetic placebo) device, a 3-cm thick wooden tool shaped and coloured as a real 8-shaped coil, and containing a bipolar electrical stimulator on the surface which comes in contact with the scalp. During real TMS, the REMP device lies over the magnetically stimulating coil, whilst during sham stimulation the position of the two coils is inverted, so that the REMP is between the real coil and the scalp. This way, both the acoustic sensation and visual impact are the same and the scalp sensation of the real TMS is synchronously reproduced by the electrical stimulator of the REMP device (Fig. 1). Here we present a series of experiments aimed to demonstrate the physical, physiological and behavioural reliability of the REMP device.

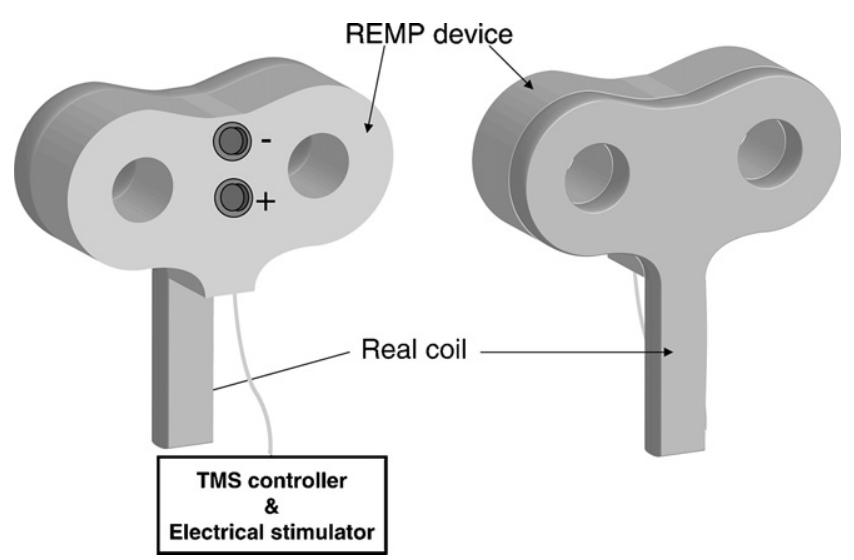

Fig. 1. Schematic drawing representing the REMP device. The REMP is firmly attached to the real coil by means of Velcro ${ }^{\circledR}$ strips passing through the holes of the two coils (not shown here). On the left side the placebo configuration is shown. For real TMS (right side) the position of the coil and of the REMP is reversed. Scalp sensations induced by real TMS are reproduced by the electrical stimulator housed within the REMP device, which is connected to the TMS and electrical stimulator controller. The visual and acoustic impact of the sham condition are the same as of the real TMS configuration.

\section{Subjects and methods}

\subsection{Subjects}

All participants were healthy volunteers who gave their written informed consent to participate in the study, which was approved by the local Ethics Committee. They were seated in a comfortable chair with their arms fully supported and were asked to report any potential adverse effect experienced during or after TMS.

\subsection{The REMP device (Fig. 1)}

The REMP device (weight about $230 \mathrm{~g}$ ) is built in compact wood, and is of the same shape and colour as the $70 \mathrm{~mm}$ eight-shaped coil produced by Magstim Co. (Whitland, Dyfed, UK). It is attached to the real coil by Velcro strips. Eventually, other coils with different shapes and materials could be easily reproduced. It has two main functions: the first one is to act as a physical spacer between the real coil and the scalp and the latter is to house a bipolar electrical stimulator within the surface which is in contact with the scalp.

The thickness of the REMP $(3 \mathrm{~cm})$ is theoretically sufficient to attenuate most of the induced electric currents reaching the brain. Indeed, the decay of the induced electrical field with distance from the coil is very steep along the axis orthogonal to the coil plane (Cincotta et al., 2005). Accordingly, previous studies indicated that TMS activates superficial neural elements at a depth lower than $3 \mathrm{~cm}$ (Roth et al., 1991; Lisanby et al., 2001).

The electrical stimulator (anode proximal to the coil's handle; interelectrodic distance $3 \mathrm{~cm}$ ) is enclosed in the middle of one surface of the REMP, so that electrodes protrude less than $1 \mathrm{~mm}$. These are round copper metal disks with a diameter of $2 \mathrm{~cm}$, aligned with the coil midline: the unusual width of the electrodes allows a less focal electrical stimulation of the scalp than that produced by commonly used smaller electrodes, making such stimulation subjectively more similar to the real TMS. The cables of the electrodes are also housed within the REMP and can be connected to the electric stimulator of the electromyographer which also controls the magnetic stimulator (Reporter, EB Neuro, Florence, Italy). In this way, electric and magnetic pulses are synchronously delivered at any desired frequency and intensity.

The following experiments were aimed to define the physical and neurophysiological reliability of the REMP device and its behavioural advantages in comparison to other available sham TMS procedures:

\subsection{Relationships between electrically- and magnetically- evoked scalp sensations}

The first step was to determine the subjective equivalence between scalp sensations evoked by the electric pulses of the REMP device with those magnetically-induced by the real TMS at different intensities of stimulation. To this 


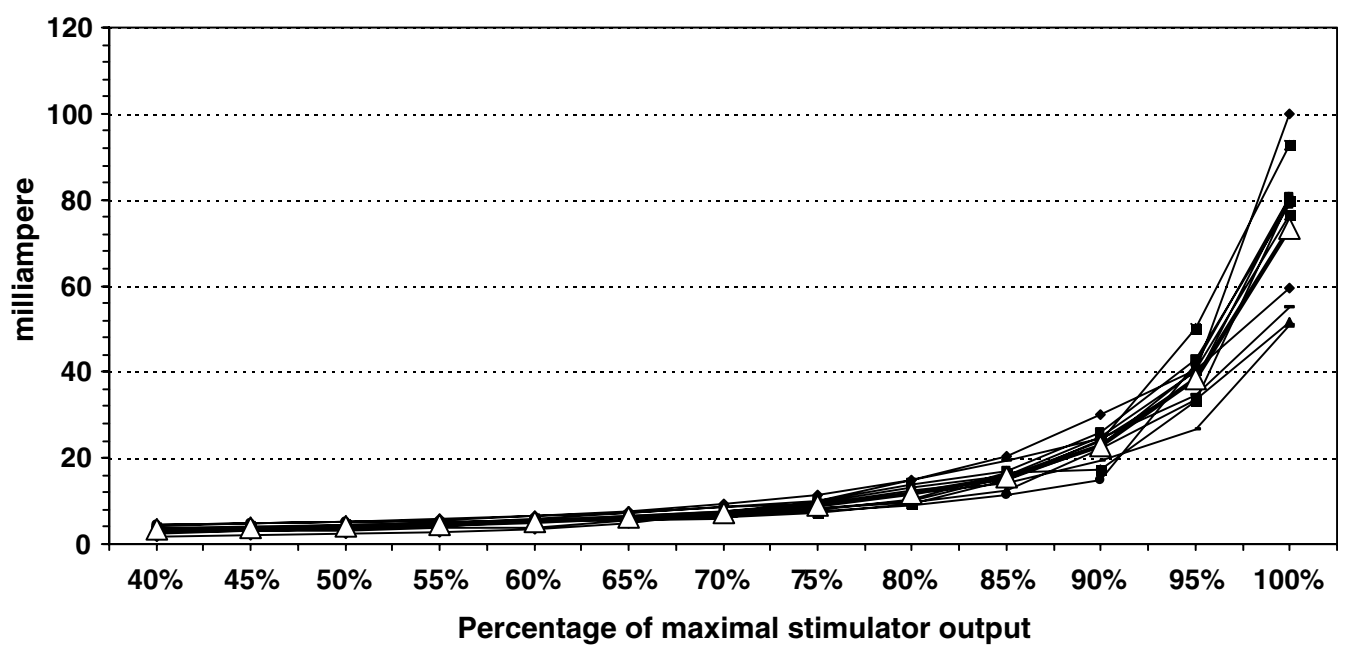

Fig. 2. Relationships between increasing intensities of TMS and current intensity required to better approximate the TMS-induced scalp sensation in 10 healthy volunteers expert on TMS. The thick line with white triangles represents the average value.

end, 10 healthy individuals, experienced with TMS procedures (mean age $34.8 \pm 7.8 \mathrm{yr}$, range $28-49 \mathrm{yr}$ ), received active biphasic TMS pulses produced by a Magstim Super Rapid stimulator (Magstim Co., Whitland, Dyfed, UK) connected to a $70-\mathrm{mm}$ eight-shaped focal coil. The coil was placed tangentially to the scalp, with the handle pointing backwards, and centred on the midline $2 \mathrm{~cm}$ posterior to the inter-aural line. A non-motor brain area was necessarily targeted to allow subjects to fully concentrate on the scalp sensation and avoid possible subjective biases due to progressively increasing muscle twitches following TMS of the motor cortex at increasing intensity. Single TMS pulses were delivered at random intensities ranging between $40 \%$ and $100 \%$ of the maximal stimulator output (MSO). Immediately after each TMS pulse, electric pulses of slightly increasing intensity were delivered to the same scalp position by the REMP device, to establish the stimulus intensity producing the closest scalp sensation to the immediately preceding TMS pulse. This was verified pulse-by-pulse by verbal answers of the subjects. The electrical stimulus was a square wave pulse with its length adjusted at $250 \mu \mathrm{sec}$ [i.e. the duration of the most relevant part of the electric field induced by the TMS pulse (see. Fig. 3)]. Finally, electrical stimulus intensities (in milliampere) were plotted against the corresponding TMS intensities (Fig. 2).

\subsection{Physical measurements}

In a successive experiment, the physical properties of monophasic and biphasic electric fields induced by real TMS, REMP-attenuated TMS, and by the $70 \mathrm{~mm}$ eightshaped sham coil were measured at increasing intensities of stimulation ranging between $10 \%$ and $100 \%$ of the MSO. As magnetic stimulators we used a Magstim Rapid with two booster modules for biphasic stimulation and a Magstim 200 for monophasic stimulation (Magstim Co.,
Whitland, Dyfed, UK). For each intensity applied, three identical responses were collected. For the condition TMS+REMP, the TMS-induced electric field was measured either with or without the concurrent electrical stimulation of the REMP device, which was adjusted according to the function extrapolated by the graph in Fig. 2.

Measurements of electric fields were carried out by a dipole probe (Tofts and Branston, 1991; Cincotta et al., 2005). Briefly, the dipole probe (Fig. 3a) consisted of two insulated wires bared at their tips and twisted together along the axis perpendicular to the plane of the coil, with a distance between the wire tips of less than $0.5 \mathrm{~mm}$. This prevented the time-varying magnetic field from inducing a voltage in the leads. The wires of the dipole probe were connected to a pre-amplifier and the TMS-induced voltages were analogue filtered $(2 \mathrm{~Hz}-20 \mathrm{kHz})$ and then digitised at a very high $\mathrm{A} / \mathrm{D}$ rate $(500 \mathrm{kHz})$ by a Micro 1401 unit (Cambridge Electronic Design, UK). For data analysis, the Signal $2{ }^{\circledR}$ software (Cambridge Electronic Design, UK) was used. The dipole probe was placed $20 \mathrm{~mm}$ underneath the centre of the junction of the test coil to simulate the approximate depth at which human motor cortex is activated by TMS (Epstein et al., 1990) (see Fig. 3a). As the dipole probe measures voltages along the line joining the two wire tips (Tofts and Branston, 1991), this line was oriented parallel to the junction of the test coil. This allowed us to focus measurements on the induced electrical field component, which plays a pivotal role in trans-synaptic activation occurring at cortical level (Rouhonen, 2003). The voltage induced in the dipole probe was measured from baseline $(0 \mathrm{mV})$ to the peak of the major deflection (Fig. 3b and c).

Finally, stimulus intensity/induced field curves were plotted for the four conditions: real TMS, TMS+REMP (with or without concurrent electrical stimulation) and Magstim sham coil. This was done both for monophasic and biphasic TMS pulses. 

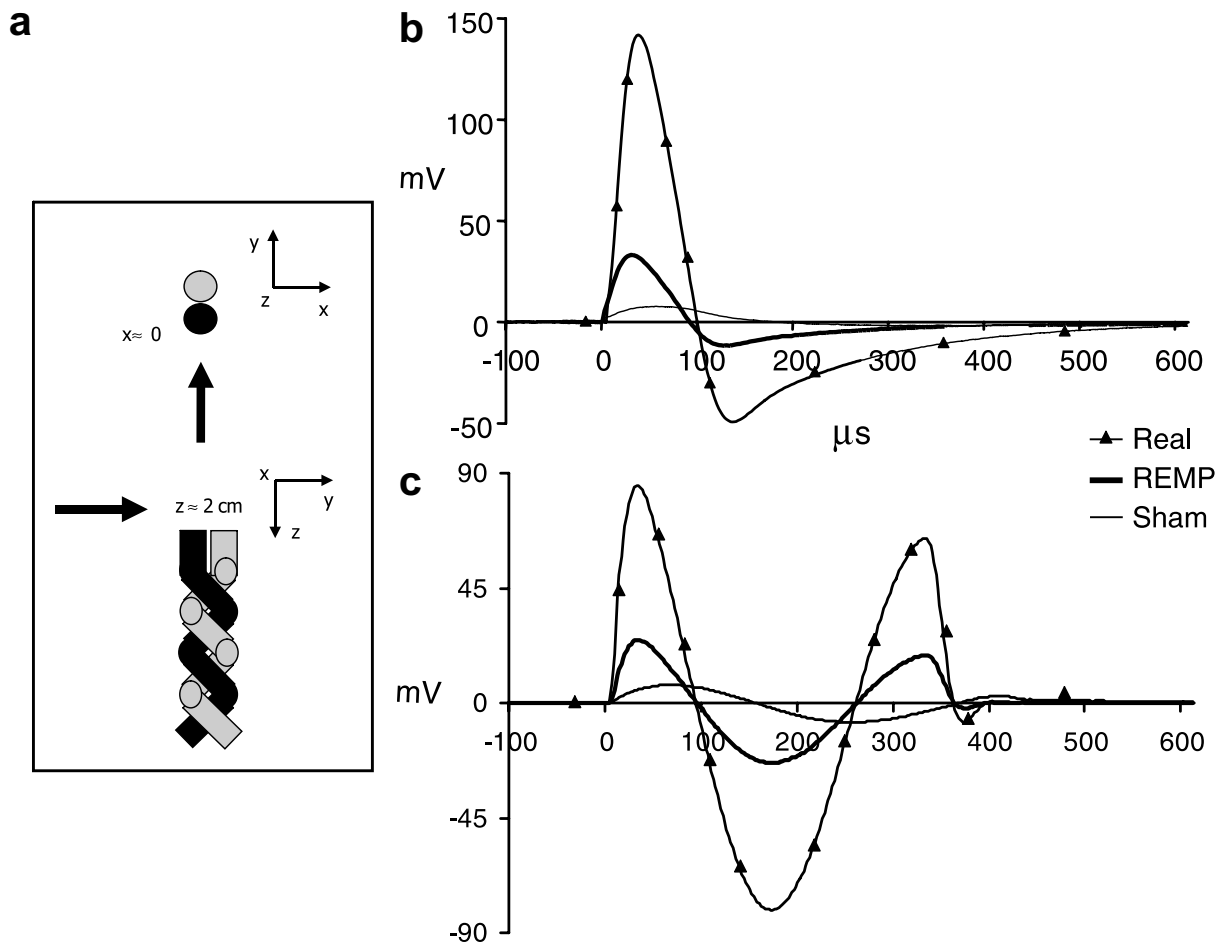

Fig. 3. (a) Scheme of the dipole probe (see text for details) used to measure electric fields induced by monophasic TMS pulses (b) and electric fields induced by biphasic TMS pulses (c) during real TMS (line with triangles), REMP-attenuated TMS (the thick line) and sham TMS with the Magstim coil (the thin line). Intensity of TMS is $60 \%$ of the MSO.

\subsection{Neurophysiological measurements}

In three out of the 10 healthy subjects mentioned above, the following neurophysiological measures were carried out, in order to exclude biologically detectable effects of real TMS+REMP device:

(a) Attempts to record motor evoked potentials from the right abductor pollicis brevis (ABP) muscle after REMP-attenuated monophasic single pulse of the left primary motor cortex delivered at $100 \%$ of the MSO. Recordings were carried out through surface electrodes at rest and during voluntary contraction of the ABP muscle, a condition in which the probability of a TMS-linked corticospinal neuronal discharge with consequent muscular activation is much higher than during target muscle relaxation (Hess et al., 1987; Rossini et al., 1999). Bandpass filtering was $20 \mathrm{~Hz}-2 \mathrm{kHz}$, with amplifiers at a maximal gain.

(b) Attempts to record compound motor action potential (cMAP) from the right APB, with the coil+REMP device placed on the volar aspect of the forearm to stimulate motor fibers of the median nerve. A total of 20 monophasic magnetic stimuli were delivered at $100 \%$ of the MSO, with different orientations of the coil handle.

(c) Since some paraesthesia-like sensations in the median nerve territory were occasionally reported after stimulations at the forearm, we also attempted to record antidromic sensory nerve action potentials (SAPs) after stimulation of the forearm as in (b). Recordings were made using ring electrodes placed over the index finger. One hundred REMP-attenuated monophasic magnetic stimuli (at $100 \%$ of the MSO) were averaged.

(d) It could not be excluded that sensory fiber activation, although subthreshold for evoking a recordable SAP, might have taken place after REMP-attenuated TMS stimuli and elaborated at cortical level through thalamic amplification, as in case of severe peripheral neuropathies (Parry and Aminoff, 1987). This mechanism, indeed, might explain awareness of paraesthesias. Hence, an additional control condition considered cortical somatosensory evoked potentials (SEPs) after stimulation of peripheral nervous fibers by REMP-attenuated TMS stimuli. Cortical SEPs were recorded in three conditions: after conventional electrical stimulation of the median nerve at the wrist, after stimulation with repetitive TMS biphasic stimuli at $65 \%$ of the MSO, and after REMP-attenuated TMS stimuli at $100 \%$ of the MSO. In the first two conditions each stimulus elicited a visible muscle twitch in thenar muscles. SEPs were obtained averaging $250-1 \mathrm{~Hz}$ responses for each condition, and recorded by electrodes placed on the contralateral hemisphere overlying the primary sensory cortex (for technical details of SEP recordings, see Rossi et al., 2005). 


\subsection{Behavioural evaluations}

This step was carried out on seven healthy subjects (four male; mean age $30.8 \pm 4.4 \mathrm{yr}$, range $28-41 \mathrm{yr}$ ) who had previously participated in several TMS experiments (TMS experts) and eight healthy subjects of comparable gender and age (4 males; mean age $29.5 \pm 4.2 \mathrm{yr}$, range 25-36 yr), with no experience of TMS procedures (TMS naïve). Each subject received, on separate days, six sequences (i.e., trials) of four stimuli (interstimulus interval ranging between 5 and $10 \mathrm{~s}$ ), including one real TMS train and three sham TMS stimuli, that is sham TMS with the coil tilted at $90^{\circ}$, sham TMS with the Magstim sham coil and sham TMS with the previously described REMP-attenuated procedures. Each sequence of stimuli, delivered on the same scalp region as in experiment 1 , was carried out with four combinations of intensity and frequency: $90 \%$ of individual resting motor threshold (RMT) of the right APB at $1 \mathrm{~Hz}$ (for $10 \mathrm{~s}$ ) and $20 \mathrm{~Hz}$ (for $1 \mathrm{~s}$ ), and $120 \%$ of RMT at $1 \mathrm{~Hz}$ (for $10 \mathrm{~s}$ ) and $20 \mathrm{~Hz}$ (for $1 \mathrm{~s}$ ). Subjects were tested and retested in different days, with the order of sequences and TMS conditions randomized and counterbalanced. After each of the four sequences of stimuli, the following question was asked to subjects: which of the four stimuli you believe was the real TMS of your brain? Hence, subjects were asked to distinguish between four categorical variables for six trials $\times$ TMS condition. Subjects were allowed to additionally comment on subjective sensations following each type of TMS.

Descriptive analysis of results was followed by KruskalWallis test to evaluate possible effects of temporal order of trials and sequences. This was carried out both for "Real" and "REMP" TMS conditions. Finally, chi-square for categorical variables was applied for each TMS intensity/frequency condition. The level of significance was set at $p<0.05$ for all tests.

\section{Results}

None of the participants reported adverse reactions during or after the experimental procedures.

\subsection{Relationships between electrically-and magnetically- evoked scalp sensations}

The graph in Fig. 2 shows that the intensity of electric pulses delivered by the REMP required to subjectively obtain a scalp sensation similar to the real TMS was constant across subjects, at least for TMS intensities ranging from $10 \%$ to $80 \%$ of the MSO. Among different regression analyses, the best approximation for these experimental data was the following logarithmic function:

$y=0.347$ const $\mathrm{e}^{0.047 x}$ where " $y$ " is the required current intensity (in milliampere), and " $x$ " is the desired TMS intensity (expressed as percentage of the MSO).

However, most of subjects qualitatively reported that higher the intensity of the TMS pulse (i.e., more than $80 \%$ ) the more diffused and more "deeper" was the scalp sensation compared to that induced by the electric pulse of the REMP device.

\subsection{Physical measurements}

Electric fields induced by monophasic or biphasic real TMS alone, by TMS+REMP device and by the Magstim sham coil are reported in Fig. 4. None of the procedures for sham TMS abolished the induced electric field as measured by the dipole probe. However, the degree of

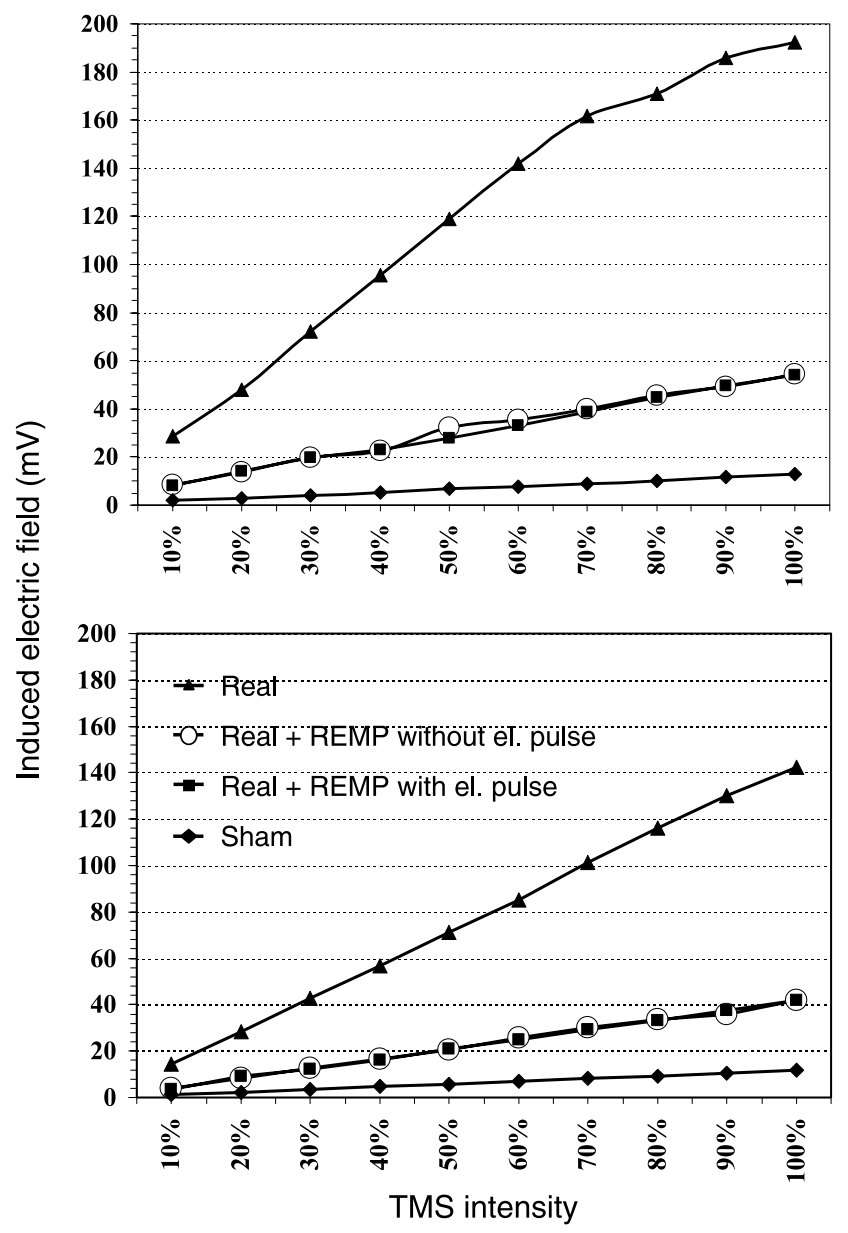

Fig. 4. Induced electric fields by single-pulse TMS at increasing intensities, as measured by the dipole probe. Lines refer to the real TMS (black triangles), to the REMP-attenuated TMS [both with (black squares) and without (white circles) concurrent electrical stimulation] and to the TMS delivered with the Magstim sham coil (black rhombs). Upper panel: monophasic stimulation. Lower panel: biphasic stimulation. In both cases, electric fields induced by REMP-attenuated TMS at $100 \%$ of the MSO correspond to a real TMS pulse of less than $25 \%$. Note that REMPattenuated TMS pulses at $80 \%$ of the MSO (i.e., the maximal intensity generally used in clinical and experimental settings) correspond to about $18 \%$ (monophasic)-22\% (biphasic) real pulses. 
attenuation of the induced electric field by the REMP device was constantly efficient at all stimulation intensities (details in the figure).

\subsection{Neurophysiological results}

By using REMP-attenuated TMS stimuli at $100 \%$ of the MSO, no motor responses were obtained after stimulation of the motor cortex in the relaxed or contracted target muscle; the same was true after peripheral stimulation. Similarly, the same REMP-attenuated TMS stimulus did not evoke detectable activation of peripheral sensory and motor fibers.

Either electric or magnetic (by the real coil) median nerve stimulation at the forearm elicited clearly detectable responses in the contralateral primary somatosensory cortex, peaking around $20 \mathrm{~ms}$ after the stimulation. In contrast, REMP-attenuated TMS stimuli failed to elicit sensory cortex activation.

\subsection{Behavioural results}

All TMS experts were able to indicate that the real TMS was the only condition in which their brain was actively stimulated, regardless of the intensity and frequency of TMS.

At a descriptive level, from $25 \%$ to $50 \%$ of the TMSnaïve subjects consistently (six times out of six trials) identified the real TMS as such in each of the four TMS conditions (Table 1), while about $40 \%$ of them (i.e., not sure + REMP of Table 1) consistently judged the "REMP" as real or were uncertain between the two. The remaining subjects identified more than a single categorical variable in the six trials (i.e., they gave different responses to the question), mainly confusing the "Real" and "REMP" TMS (Fig. 5). Moreover, none thought to have received a real brain stimulation after TMS with the Magstim sham coil or with the coil tilted at $90 \%$. The pattern of categorical variables identification was always significantly different ( $p<0.0001$ chi-square test) across the four TMS conditions $(1 \mathrm{~Hz}$ at $90 \%, 1 \mathrm{~Hz}$ at $120 \%, 20 \mathrm{~Hz}$ at $90 \%$, and $20 \mathrm{~Hz}$ at $120 \%$ of RMT).

The order of trials and sequences within each trial did not significantly influence the answers in any TMS condition (Kruskal-Wallis test always n.s.). This suggests that the identification of the real or REMP-attenuated TMS

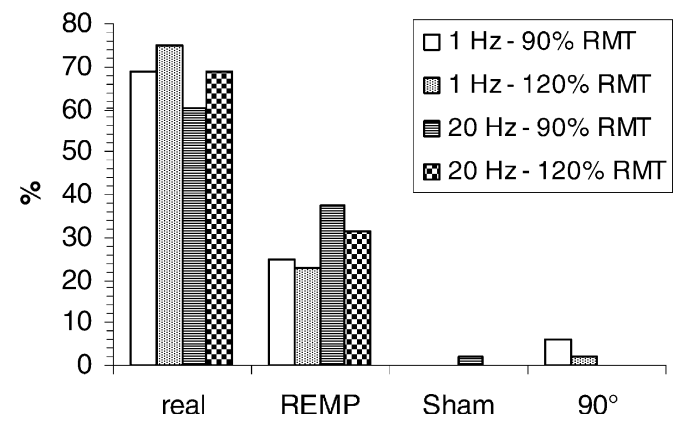

Fig. 5. Bars represent the percentage of responses given to the question "which of the four stimuli you believe is the real TMS?" (pooled subjects). Note that none identified the TMS given with the commercially available sham coil as real and that tilting the coil at $90^{\circ}$ led to a similar result. The "Real" TMS was correctly identified from $60-75 \%$ of the sequences in the four TMS conditions. The REMP TMS was to identified as real TMS in $25-40 \%$ of sequences. Statistics are in the text.

was not influenced either by "first impact" or recency effects.

\section{Discussion}

There is general agreement that an ideal sham TMS should fulfil the following criteria: absence of biological effects on the cortex, same visual impact and position of the real coil on the scalp, and same acoustic and sensory afferent sensations (Loo et al., 2000; Lisanby et al., 2001; Sommer et al., 2006).

Tilting the stimulating coil at less than $90^{\circ}$ is biologically active on the brain (Lisanby et al., 2001). Both sham TMS with the coil at $90^{\circ}$, or keeping the coil at a distance from the scalp, fail to evoke scalp sensations, making it easy, even for TMS-naïve subjects, to realize that they are not receiving real stimulation. The lack of clear-cut TMSlinked scalp sensations, besides the mere coil-scalp contact, is again a failure of the commercially available Magstim sham coil, as well as of the sandwich verum/sham coil complex (Sommer et al., 2006) and of the double-coil-complex introduced by Rouhonen and coworkers (2000). Additionally, intensity and sound location of the acoustic artefact of the Magstim sham coil markedly differ from those of the real TMS. Therefore, none of these available sham procedures are fully similar to a real TMS application. This aspect is clearly highlighted by the current behavioural results: indeed, neither expert nor naïve subjects identified

Table 1

Number of naïve TMS subjects (total sample 8 ) who gave always the same answer (6/6 trials) following the question: which of the four TMS stimuli you believe was the real TMS?

\begin{tabular}{llll}
\hline Type of TMS & Answers & & Not sure (between "Real" and "REMP") \\
\cline { 2 - 3 } & "Real" as real & 2 & 1 \\
\hline $1 \mathrm{~Hz}, 90 \%$ of RMT & 2 & 2 & 1 \\
$1 \mathrm{~Hz}, 120 \%$ of RMT & 4 & 2 & 1 \\
$20 \mathrm{~Hz}, 90 \%$ of RMT & 3 & 2 & 1 \\
$20 \mathrm{HZ}, 120 \%$ of RMT & 3 & & 1 \\
\hline
\end{tabular}


the "coil at $90^{\circ}$ " or the "Magstim sham coil" as a real TMS intervention (Fig. 5), an expected but not yet formally tested result.

In a previous study (Okabe et al., 2003), a more realistic sham procedure considered the repetitive electrical stimulation of the scalp, coupled with an inactive coil placed on the vertex and an additional discharging coil "near to the subject"; however, despite similar scalp sensations during real and sham TMS, these two conditions differed in terms of visual impact (two coils used for sham and a single coil for real TMS) and absence of acoustic bone transmission of the discharging coil.

The "goodness of sham" of the REMP device might even have been underestimated behaviourally, taking into account that our subjects were asked to discriminate the different TMS types immediately after each sequence of four stimuli. In keeping with this view, most TMS-naïve individuals commented that undergoing a REMP-attenuated or a real TMS application with a longer time interval in between (as in case of a clinical cross-over, placebo-controlled, design study), they would not be able to distinguish between the two.

In the present study, physical measurements indicate that the REMP device is able to significantly attenuate the induced electric currents in the brain following monophasic and biphasic TMS trains, both at low and high frequency $(1 \mathrm{~Hz}$ and $20 \mathrm{~Hz})$ and intensities $(90 \%$ and $120 \%$ of the RMT) of stimulation. Namely, currents induced by REMP-attenuated TMS at $60 \%$ and $100 \%$ MSO were lower than currents induced by real TMS at about $15 \%$ and $20 \%$ MSO, respectively (Fig. 4). For several reasons, including safety (Wassermann, 1998) and subject comfort and compliance, real rTMS is usually delivered at intensities lower than $80 \%$ MSO. Hence, although the electric field induced by the REMP device is higher than that produced by the commercially available sham coil, the present data indicate that REMP-induced electric field attenuation is sufficient to prevent biologically active currents reaching the brain in most, if not all, subjects. Failure of high-intensity REMP-attenuated transcranial and peripheral magnetic stimuli to evoke motor responses and the absence of cortical SEP and peripheral sensory nerve potentials following median nerve stimulation by the REMP device strongly support this view. In any case, the thickness of the REMP device could be easily increased in order to make this even less likely.

Scalp sensations induced by the electric pulses of the REMP are generally subjectively different by those induced by the real TMS, especially in case of higher intensities of stimulation. This is clearly indicated by the answers of subjects expert of TMS. In naïve individuals, however, this difference can be reduced by using larger electrodes (Fig. 1) than those utilized for conventional neurophysiological testing. Among scalp sensations, it should be considered that the temperature of the real coil increases with longer trains of stimulation. Although this did not occur with the brief stimulations utilized in the current study, this rep- resents a possible bias, that can be easily solved switching the coil before heating.

It seems unlikely that the electric pulses of the REMP device are biologically active on the underlying cortex. Electric stimulation of the brain through square wave pulses needs enormously higher current intensities than those delivered by the REMP device (Rossini et al., 1994).

A possible limitation of the REMP device is that it may prevent current spread towards the skull base (and consequent ipsilateral facial nerve activation), which are likely to occur with real TMS targeting scalp areas close to the temporal region. As previously suggested (Sommer et al., 2006), this bias could be easily overcome by an additional concurrent electrical stimulation of the facial nerve trunk ipsilateral to the REMP-attenuated TMS. An unsolved shortcoming of the REMP device is that targeting the motor cortex, no muscular twitches can be evoked, even during voluntary contraction and at an intensity of $100 \%$ MSO. However, this shortcoming is also present with all current placebo TMS procedures.

In conclusion, the "goodness of sham" of the REMP device is physically and neurophysiologically relevant, especially when targeting brain regions where the real TMS does not spread to the ipsilateral facial nerve. When applied on subjects naïve of TMS, as in the case of patients candidate for neuromodulatory rTMS treatment, it has several behavioural advantages in comparison to the other available sham TMS procedures. Finally, it is extremely low cost and can be easily adapted to all commercially available coils.

\section{Acknowledgments}

Authors thank Drs G. Greco, A. Di Rollo, A. De Capua, and A. Borgheresi for experimental help and constructive criticism.

This research was supported by a Project grant from the Ministero della Salute RF 2005/56.

The Center of Florence was also supported by a grant from "Ente Cassa di Risparmio di Firenze", Florence, Italy.

\section{References}

Cincotta M, Borgheresi A, Jung P, Balestrieri F, Giovannelli F, Zaccara $\mathrm{G}$, et al. Physical interaction between induced electrical fields can have substantial effects on neuronal excitation during simultaneous TMS of two brain areas. Clin Neurophysiol 2005;116:1733-42.

Epstein CM, Schwartzberg DG, Davey KR, Sudderth DB. Localizing the site of magnetic brain stimulation in humans. Neurology 1990;40:666-70.

Fregni F, Pascual-Leone A. Transcranial magnetic stimulation for the treatment of depression in neurologic disorders. Curr Psychiatry Rep 2005; 7:381-90.

Hallett M. Transcranial magnetic stimulation and the human brain. Nature 2000;406:147-50.

Hess CW, Mills KR, Murray NMF. Responses in small hand muscles from magnetic stimulation of the human brain. J Physiol (Lond) 1987;388:397-419. 
Hoffman RE, Cavus I. Slow transcranial magnetic stimulation, long-term depotentiation, and brain hyperexcitability disorders. Am J Psychiatry 2002;159:1093-102.

Lisanby SH, Gutman D, Luber B, Schroeder C, Sackeim HA. Sham TMS: intracerebral measurement of the induced electrical field and the induction of motor-evoked potentials. Biol Psychiatry 2001;49: $460-3$.

Loo CK, Taylor JL, Gandevia SC, McDarmont BN, Mitchell PB, Sachdev PS. Transcranial magnetic stimulation (TMS) in controlled treatment studies: are some "sham" forms active? Biol Psychiatry 2000;47:325-31

Okabe S, Ugawa Y, Kanazawa I. Effectiveness of rTMS on Parkinson's Disease Study Group. 0.2-Hz repetitive transcranial magnetic stimulation has no add-on effects as compared to a realistic sham stimulation in Parkinson's disease. Mov Disord 2003;18:382-8.

Parry GJ, Aminoff MJ. Somatosensory evoked potentials in chronic acquired demyelinating peripheral neuropathy. Neurology 1987;37: 313-6.

Pascual-Leone A, Walsh V, Rothwell J. Transcranial magnetic stimulation in cognitive neuroscience - virtual lesion, chronometry, and functional connectivity. Curr Opin Neurobiol 2000;10:232-7.

Rossi S, Rossini PM. TMS in cognitive plasticity and the potential for rehabilitation. Trends Cogn Sci 2004;8:273-9.

Rossi S, Bartalini S, Ulivelli M, Mantovani A, Di Muro A, Goracci A, et al. Hypofunctioning of sensory gating mechanisms in patients with obsessive-compulsive disorder. Biol Psychiatry 2005;57:16-20.

Rossini PM, Rossi S. Transcranial magnetic stimulation: diagnostic, therapeutic, and research potential. Neurology, 2006, in press.

Rossini PM, Barker AT, Berardelli A, Caramia MD, Caruso G, Cracco $\mathrm{RQ}$, et al. Non-invasive electrical and magnetic stimulation of the brain, spinal cord and roots: basic principles and procedures for routine clinical application. Report of an IFCN committee. Electroencephalogr Clin Neurophysiol 1994;91:79-92.

Rossini PM, Rossi S, Pasqualetti P, Tecchio F. Corticospinal excitability modulation to hand muscles during movement imagery. Cereb Cortex 1999;9:161-7.

Roth BJ, Saypol JM, Halett M, Cohen LG. A theoretical calculation of the electric field induced in the cortex during magnetic stimulation. Electroenceph Clin Neurophysiol 1991;81:47-56.

Rouhonen J. Background physics for magnetic stimulation. Suppl Clin Neurophysiol 2003;58:3-12.

Rouhonen J, Ollikainen M, Nikouline V, Virtanen J, Ilmoniemi RJ. Coil design for real and sham transcranial magnetic stimulation. IEEE Trans Biomed Eng 2000;47:145-8.

Sommer J, Jansen A, Drager B, Steinstrater O, Breitenstein C, Deppe M, et al. Transcranial magnetic stimulation - a sandwich coil design for a better sham. Clin Neurophysiol 2006;117:440-6.

Strafella AP, Ko JH, Monchi O. Therapeutic application of transcranial magnetic stimulation in Parkinson's disease: the contribution of expectation. Neuroimage 2006;31:1666-72.

Tofts PS, Branston NM. The measurement of electric field and the influence of surface charge in magnetic stimulation. Electroencephalogr Clin Neurophysiol 1991;81:238-9.

Walsh V, Cowey A. Transcranial magnetic stimulation and cognitive neuroscience. Nat Rev Neurosci 2000;1:73-9.

Wasserman EM, Lisanby SH. Therapeutic application of repetitive transcranial magnetic stimulation: a review. Clin Neurophysiol 2001;112:1367-77.

Wassermann EM. Report on risk and safety of repetitive transcranial magnetic stimulation (rTMS): suggested guidelines from the International Workshop on Risk and Safety of rTMS. June 5-7 1996. Electroencephalogr Clin Neurophysiol 1998;108:1-16. 